\title{
Improved Multiobjective Harmony Search Algorithm with Application to Placement and Sizing of Distributed Generation
}

\author{
Wanxing Sheng, ${ }^{1}$ Ke-yan Liu, ${ }^{1}$ Yunhua Li, ${ }^{2}$ Yuan Liu, ${ }^{2}$ and Xiaoli Meng' \\ ${ }^{1}$ Power Distribution Department, Electric Power Research Institute of China, No. 15, Xiaoying East Road, \\ Qinghe, Haidian District, Beijing 100192, China \\ ${ }^{2}$ School of Automation Science and Electrical Engineering, Beihang University, No. 37, Xuanyuan Road, \\ Haidian District, Beijing 100191, China \\ Correspondence should be addressed to Ke-yan Liu; liukeyan@epri.sgcc.com.cn and Yunhua Li; yhli@buaa.edu.cn
}

Received 27 January 2014; Accepted 22 March 2014; Published 10 April 2014

Academic Editor: Her-Terng Yau

Copyright (C) 2014 Wanxing Sheng et al. This is an open access article distributed under the Creative Commons Attribution License, which permits unrestricted use, distribution, and reproduction in any medium, provided the original work is properly cited.

To solve the comprehensive multiobjective optimization problem, this study proposes an improved metaheuristic searching algorithm with combination of harmony search and the fast nondominated sorting approach. This is a kind of the novel intelligent optimization algorithm for multiobjective harmony search (MOHS). The detailed description and the algorithm formulating are discussed. Taking the optimal placement and sizing issue of distributed generation (DG) in distributed power system as one example, the solving procedure of the proposed method is given. Simulation result on modified IEEE 33-bus test system and comparison with NSGA-II algorithm has proved that the proposed MOHS can get promising results for engineering application.

\section{Introduction}

Multiobjective optimization is concerned with mathematical optimization problems involving more than one objective to be optimized simultaneously, and usually these objectives are in conflict with each other. Optimal decisions need to be taken in the presence of tradeoffs between two or more conflicting objectives. It has been applied in many fields of engineering. In [1], a steepest descent method was proposed in the scheduling of a mobile sensor's coverage time among a set of points of interest for optimal tradeoff among different performance goals with regard to the distributions of per point of interest coverage times and exposure times, the entropy and energy efficiency of sensor movement. In [2], in order to solve the optimization problem to minimize energy consumption and reduce total completion time of a single machine, a multiobjective genetic algorithm is proposed to obtain an approximate set of nondominated alternatives. And several rules and heuristics are utilized to increase the speed of the proposed algorithm.

There are two approaches in solving the multiobjective optimization problem: (1) finding optimal solution by using a weighted sum approach; (2) finding optimal solution based on Pareto-optimal front. The first approach aims to solve the multiobjective problem through using the weighting factors to transform the multiple objectives into one objective. In this approach, how to define the weighting factors among the multiple objectives is a difficult problem. And the weighting factors need to be tuned to comprehensively express the optimization relation among the multiple objectives, such as the work carried out in [3-6].

The second approach aims to optimize all objective functions simultaneously based on nondominated solutions. In general, a Pareto front which is composed with all candidate solutions for the multiobjective optimization will be achieved at the end of the second approach. And the solution got by the first approach would be one of the nondominated solutions found by the second approach. In [7], in order to avoid the difficulty to assign fitness to individuals in evolutionary algorithm, a novel coevolutionary technique named multiple populations for multiple objectives was proposed. It solved multiple objective problems by letting each population correspond with only one objective. In addition, it also used an external shared archive for different populations to exchange search information and by using two novel designs to enhance the performance. 
Harmony search (HS) [8] is a metaheuristic algorithm inspired by the improvisation process of musician. In the HS algorithm, each musician which represents decision variable plays a note, namely, generates a value, to obtain the best harmony which means global optimum. The standard HS performs well in finding optimum but it encounters problems when facing multiobjective optimization problem. The main drawback of standard HS is that it generates only one solution at the end of search, and the optimization solution critically depends on the selected weights for each cost function. A multiobjective harmony search (MOHS) algorithm for optimal power flow was investigated in [9]. Fast elitist nondominated sorting and crowding distance had been used to find and manage the Pareto optimal front. In [10], a version of MOHS was introduced to solve the two-dimensional optimization problem. However, in that algorithm, when a new harmony is generated, the harmony memory had to be reordered to determine whether the new generated harmony replaces an existing one or not. It takes a huge amount of time as there are dozens of new harmonies generated in each iteration. In order to deal with above problem, an improved MOHS was proposed to combine the standard HS with the fast nondominated approach to find the Pareto-optimal front of feasible solutions in this paper. And the best compromised solution can be extracted from Pareto-optimal front through a method based on the fuzzy theory.

In this paper, the proposed MOHS is utilized to solve the multiobjective optimization problem for the integration of distributed generation (DG). DG can effectively improve the stability, power quality, and energy efficiency in addition to the environment protection [11]. Taking full benefit of DG units in distribution power system will depend on the detail position and capacity of DG units in the system, the DG placement and sizing become an optimal planning problem to solve in power utility. In addition, along with the strict demands of distribution power system operation safely, the optimal placement and sizing of DG problem no longer only focus on the single objective such as minimum power loss or investment cost consideration, but it also takes other objectives like voltage quality and operation stability into consideration [12]. In recent literatures, the DG allocation is optimized to minimize power loss of the system, enhance reliability, and improve voltage profile with a novel approach based on dynamic programming in [13]. Time varying load was also applied and cost/benefit forms were used in the optimization to reach pragmatic results. In [14], a new optimization problem was proposed to determine the maximum DG penetration level with the consideration of harmonic and protection coordination limits. In this paper, three objectives as minimum power loss, minimum voltage deviation, and maximal voltage stability margin are taken into account for the optimal placement and sizing of DG.

The rest of the paper is organized as follows. Section 2 presents the detailed procedure of the proposed MOHS algorithm. The application of MOHS for optimal placement and sizing of DG is formed in Section 3. Numerical optimization results and comparisons with NSGA-II are provided in Section 4 in IEEE 33-bus test case using the proposed MOHS algorithm. Section 5 summarizes the main contributions and conclusions.

\section{Formulation of Multiobjective Optimization Problem}

In a general formulation, a multiobjective optimization problem with $m$ objectives and $n$ controlling variables can be described as follows:

$$
\begin{array}{ll}
\min & y=F(x)=\left(f_{1}(x), f_{2}(x), \ldots, f_{m}(x)\right)^{T}, \\
\text { s.t. } & g_{i}(x) \leq 0, \quad i=1,2, \ldots, q, \\
& h_{j}(x)=0, \quad j=1,2, \ldots, p,
\end{array}
$$

where $x=\left(x_{1}, x_{2}, \ldots, x_{n}\right) \in X \subset R^{n}$ is the $n$-dimension decision vector, $X$ is the $n$-dimension decision space, and $y=\left(y_{1}, y_{2}, \ldots, y_{m}\right) \in Y \subset R^{m}$ is the $m$-dimension objective vector. $F(x)$ is the mapping function from the decision space to objective space. $g_{i}(x) \leq 0$ is the $i$ th inequality constraint and $h_{j}(x)=0$ is the $j$ th inequality constraint. $q$ and $p$ are the number of inequality and equality constraints, respectively.

In order to solve the multiobjective optimization problem using the proposed MOHS algorithm, several definitions should be clarified as follows.

Definition 1. A solution $x_{1}$ is said to dominate $x_{2}$ (denoted by $x_{1} \prec x_{2}$ ) if and only if

$$
\begin{aligned}
& \forall i \in\{1,2, \ldots, m\}: f_{i}\left(x_{1}\right) \leq f_{i}\left(x_{2}\right) \wedge \\
& \exists j \in\{1,2, \ldots, m\}: f_{j}\left(x_{1}\right)<f_{j}\left(x_{2}\right) .
\end{aligned}
$$

Definition 2. For $S=\left\{x_{i}, i=1, \ldots, n\right\}$, solution $x$ is said to be a nondominated solution (Pareto solution) of set $S$ if $x \in S$, and there is no solution $x^{\prime} \in S$ for which $x^{\prime}$ dominates $x$.

Definition 3. Assume that set $P$ contains all nondominated solutions of $S$; then $P F=\left\{v \mid v=\left[f_{1}(x), f_{2}(x), \ldots\right.\right.$, $\left.\left.f_{m}(x)\right]^{T}, x \in P\right\}$ is a Pareto front of set $S$.

\section{Proposed MOHS Algorithm}

3.1. Overview of HS. The standard HS algorithm is based on the natural musical process which searches for perfect state of harmony. Its steps include parameters initialization, initializing the harmony memory (HM), and iterations of improvising a new harmony from the harmony memory and updating harmony memory. The HS algorithm does not require initial values for the decision variables and uses a stochastic random search. In general, the HS algorithm works as follows $[8,10]$.

Step 1. Define the objective function and decision variables. Input the system parameters and the boundaries of the decision variables.

The HS algorithm parameters are specified in this step. They are the harmony memory size (HMS) or the number of solution vectors in harmony memory, the harmony memory 
considering rate (HMCR), the distance bandwidth (bw), the pitch adjusting rate (PAR), and the number of improvisations $(K)$ or stopping criterion, where $K$ is the same as the total number of function evaluations.

Step 2. Initialize the harmony memory (HM). The harmony memory is a memory location where all the solution vectors (sets of decision variables) are stored. The initial harmony memory is randomly generated.

Step 3. Improvise a new harmony from the harmony memory. Generating a new harmony $x^{\text {new }}$ is called improvisation, which is based on 3 rules: memory consideration, pitch adjustment, and random selection. First of all, a uniform random number $r$ is generated in the range $[0,1]$. If $r$ is less than the HMCR, the decision variable $x_{i}^{\text {new }}$ is generated by the memory consideration; otherwise, $x_{i}^{\text {new }}$ is obtained by a random selection. Then, each decision variable will undergo a pitch adjustment with a probability of PAR if it is produced by the memory consideration. The pitch adjustment rule is given as follows:

$$
\begin{aligned}
& x_{i}^{\text {new }}=x_{i}^{\text {new }}+r \times \text { bw } \\
& x^{\text {new }}=\left(x_{1}^{\text {new }}, x_{2}^{\text {new }}, \ldots, x_{N}^{\text {new }}\right) .
\end{aligned}
$$

Step 4. Update harmony memory. After generating a new harmony vector $x^{\text {new }}$, the harmony memory will be updated. If the fitness of the improvised harmony vector is better than that of the worst harmony, the worst harmony in the HM will be replaced with $x^{\text {new }}$ and become a new member of the HM.

Step 5. Repeat Steps 3 and 4 until the stopping criterion (maximum number of improvisations, $K$ ) is met.

3.2. MOHS Algorithm. In order to solve the comprehensive multiobjective optimization problem, the fast nondominated sorting approach described in NSGA-II [15] is employed in HS. In the proposed MOHS, an archive [16] is maintained which contains the representation of nondominated harmonies. The details of proposed MOHS algorithm for optimal placement and sizing of DG are described as follows.

Step 1 (initialize parameters). Initializing parameters includes harmony memory size (HMS), archive size (AS), and harmony memory considering rate (HMCR).

Step 2 (initialize harmonies in HM). Initialize the HM randomly within the boundaries of independent variables, and the initialized HM can be described as follows:

$$
\mathrm{HM}=\left[\begin{array}{cccc}
x_{1}^{1} & x_{2}^{1} & \cdots & x_{n}^{1} \\
x_{1}^{2} & x_{2}^{2} & \cdots & x_{n}^{2} \\
\vdots & \vdots & \cdots & \vdots \\
x_{1}^{\mathrm{HMS}} & x_{2}^{\mathrm{HMS}} & \cdots & x_{n}^{\mathrm{HMS}}
\end{array}\right]
$$

The first row in HM composes the first harmony, and so on and so forth for the rest of rows in HM.
Step 3 (generate the archive). (1) Calculate the power flow of distribution power system according to pitches of each harmony in HM, and compute each objective function value.

(2) Rank the harmonies in HM through fast nondominated sorting approach, and then the nondominated harmonies with rank 1 are moved to archive; harmonies with other ranks remain in HM.

Step 4 (generate new harmonies). (1) Select a harmony $X^{N d}$ randomly from the archive and another harmony $X^{d}$ randomly from the HM.

(2) A new harmony is generated from $X^{N d}$ and $X^{d}$ as follows:

$$
\begin{aligned}
x_{i}^{\mathrm{New}}= & x_{i}^{N d}+\operatorname{rand}(-1,1) \\
& \times\left(x_{i}^{N d}-x_{i}^{d}\right) \quad \text { if rand }(0,1)<\operatorname{HMRC} \\
x_{i}^{\mathrm{New}}= & x_{i}^{\min }+\operatorname{rand}(0,1) \\
& \times\left(x_{i}^{\max }-x_{i}^{\min }\right) \quad \text { if } \operatorname{rand}(0,1) \geq \mathrm{HMRC} \\
& \quad i=1,2,3, \ldots, N H,
\end{aligned}
$$

where $x_{i}^{\mathrm{New}}$ is the $i$ th pitch in the new harmony, $x_{i}^{N d}$ is the $i$ th pitch in $X^{N d}, x_{i}^{d}$ is the $i$ th pitch in $X^{d}, x_{i}^{\max }$ and $x_{i}^{\min }$ are the upper and lower boundaries of $i$ th pitch, and $N H$ is the number of pitches in a harmony. If a new pitch is beyond the boundary, it will be set as the boundary value.

(1) Repeat Steps 4(1) and 4(2) for HMS times.

(2) Calculate the power flow of distribution power system according to pitches of each new generated harmony, and compute each objective function value.

Step 5 (update the archive and HM). Combine the current archive, HM and new generated harmonies together, and then sort the gathered harmonies using fast nondominated sorting approach. The harmonies with rank 1 are moved to archive. If the number of harmonies with rank 1 is larger than AS, for the sake of diversity preservation, the crowdedcomparison operator [15] is introduced to select the better harmonies to constitute archive. Then harmonies with other ranks remain in HM. If the number of harmonies in $\mathrm{HM}$ is less than HMS, in order to keep diversity of HM, the rest of HMS is filled with appropriate number of randomly generated harmonies.

Step 6 (check for the stopping criterion). If the maximum number of iterations is met, turn to Step 7; otherwise, turn to Step 4 .

Step 7 (obtain the best compromised solution). The harmonies in archive compose the Pareto-optimal front of feasible solutions. The best compromise solution is extracted from archive based on the fuzzy theory [17]. The details can be described as follows.

(1) Search through all solutions; the $F_{i}^{\min }$ and $F_{i}^{\max }$ corresponding to $i$ th objective function can be found. 
(2) The membership function value $\tau_{i}^{k}$ of $i$ th objective function $F_{i}$ for the solution $k$ is defined as

$$
\tau_{i}^{k}= \begin{cases}1 & F_{i} \leq F_{i}^{\min } \\ \frac{F_{i}^{\max }-F_{i}}{F_{i}^{\max }-F_{i}^{\min }} & F_{i}^{\min }<F_{i}<F_{i}^{\max } \\ 0 & F_{i} \geq F_{i}^{\max },\end{cases}
$$

where $F_{i}^{\max }$ and $F_{i}^{\min }$ are the maximum and minimum of $i$ th objective function among all nondominated solutions, respectively. The above equation provides a measure of the degree of satisfaction for each objective function for a particular solution.

(3) The corresponding membership function $\tau^{k}$ for each solution $k$ in Pareto solution set is calculated as follows:

$$
\tau^{k}=\frac{\sum_{i=1}^{N_{\mathrm{obj}}} \tau_{i}^{k}}{\sum_{k=1}^{M} \sum_{i=1}^{N_{\mathrm{obj}}} \tau_{i}^{k}}
$$

where $M$ is the number of Pareto solutions and $N_{\text {obj }}$ is the number of optimization objectives.

(4) The best compromised solution is the one achieving the maximum of $\tau^{k}$.

The overall procedures of the proposed MOHS algorithm are illustrated in Figure 1.

\section{Practical Example}

\subsection{Problem Formulation for Optimal Placement and Sizing of DG}

4.1.1. Objective Functions. The integration of DG brings benefits to distribution power system on many aspects such as power quality improvement and line loss reduction. In order to reflect the influence of DG integration to economic performance, power quality, and operation stability, in this paper, minimum power loss, minimum voltage deviation, and maximal voltage stability margin are taken into consideration as objectives for optimal placement and sizing of DG.

Minimization of Power Loss. The first objective is to minimize system line losses after DG injection into distribution network. This objective can be expressed by the following function as

$$
F_{1}(x)=\sum_{(i, j) \in B} g_{i j}\left(V_{i}^{2}+V_{j}^{2}-2 V_{i} V_{j} \cos \theta_{i j}\right),
$$

where $B$ is the set of branches of network, and $(i, j) \in B$ means $(i, j)$ are two buses of a branch. $V_{i}$ and $V_{j}$ are voltage magnitudes of bus $i$ and $j . g_{i j}$ is the conductance between bus $i$ and $j . \theta_{i j}$ is the phase angle difference between nodal phase angles $\theta_{i}$ and $\theta_{j}$.
Minimization of Voltage Deviation. The second objective is to minimize the voltage deviation between nodal voltage and specified voltage magnitude

$$
F_{2}(x)=\sum_{i=0}^{n}\left(\frac{V_{i}-V_{i}^{\mathrm{spec}}}{V_{i}^{\max }-V_{i}^{\min }}\right)^{2}
$$

where $V_{i}$ is the voltage magnitude at $i$ th bus and $V_{i}^{\text {spec }}$ is the specified voltage magnitude. $V_{i}^{\max }$ and $V_{i}^{\min }$ are the upper limit and lower limit at $i$ th bus, respectively.

Maximization of Voltage Stability Margin. The third objective is to maximize steady-state voltage stability margin. A fast indicator of voltage stability, $L$-index, is chosen as the indicator for voltage stability index. $L$-index was presented by Kessel and Glavitsch [18] and developed by Jasmon and Lee [19]. The $L$-index of branch $i j$ can be expressed as follows:

$$
L_{i j}=\frac{4\left[\left(P_{j} X_{i j}-Q_{j} R_{i j}\right)^{2}+\left(P_{j} R_{i j}+Q_{j} X_{i j}\right) V_{i}^{2}\right]}{V_{i}^{4}} .
$$

The branch voltage will not be stable if the value of $L_{i j}$ is close to 1 , so the most instable branch in voltage stability perspective can be expressed as

$$
L=\max \left(L_{i j}\right), \quad(i, j) \in B
$$

where $L$ is the system voltage stability indicator. The steadystate voltage stability margin maximization can be further expressed as

$$
\max (1-L) \Longrightarrow \min F_{3}(x)=\min L
$$

4.1.2. Constraints. The variables in distribution power system with DG integration should satisfy the equality constraints of power flow and the inequality constraints include voltage limits of load bus and thermal limits of branch. In addition, the integration of DG is under constraints including DG output limits and penetration constraints of distribution power system.

Power flow equations describe the relationships among status variables in distribution power system operation. They are used as equality constraints as follows:

$$
\begin{gathered}
P_{\mathrm{DG} i}-P_{d i}=V_{i} \sum_{j=1}^{N} V_{j}\left(G_{i j} \cos \theta_{i j}+B_{i j} \sin \theta_{i j}\right), \\
Q_{\mathrm{DG} i}-Q_{d i}=V_{i} \sum_{j=1}^{N} V_{j}\left(G_{i j} \sin \theta_{i j}-B_{i j} \cos \theta_{i j}\right),
\end{gathered}
$$

where $P_{\mathrm{DG} i}$ and $Q_{\mathrm{DG} i}$ are active and reactive generation outputs, whereas $P_{d i}$ and $Q_{d i}$ are active and reactive loads at bus $i$, respectively, $G_{i j}$ and $B_{i j}$ are real and imaginary parts of nodal admittance matrix, respectively, and $N$ is the number of buses. 


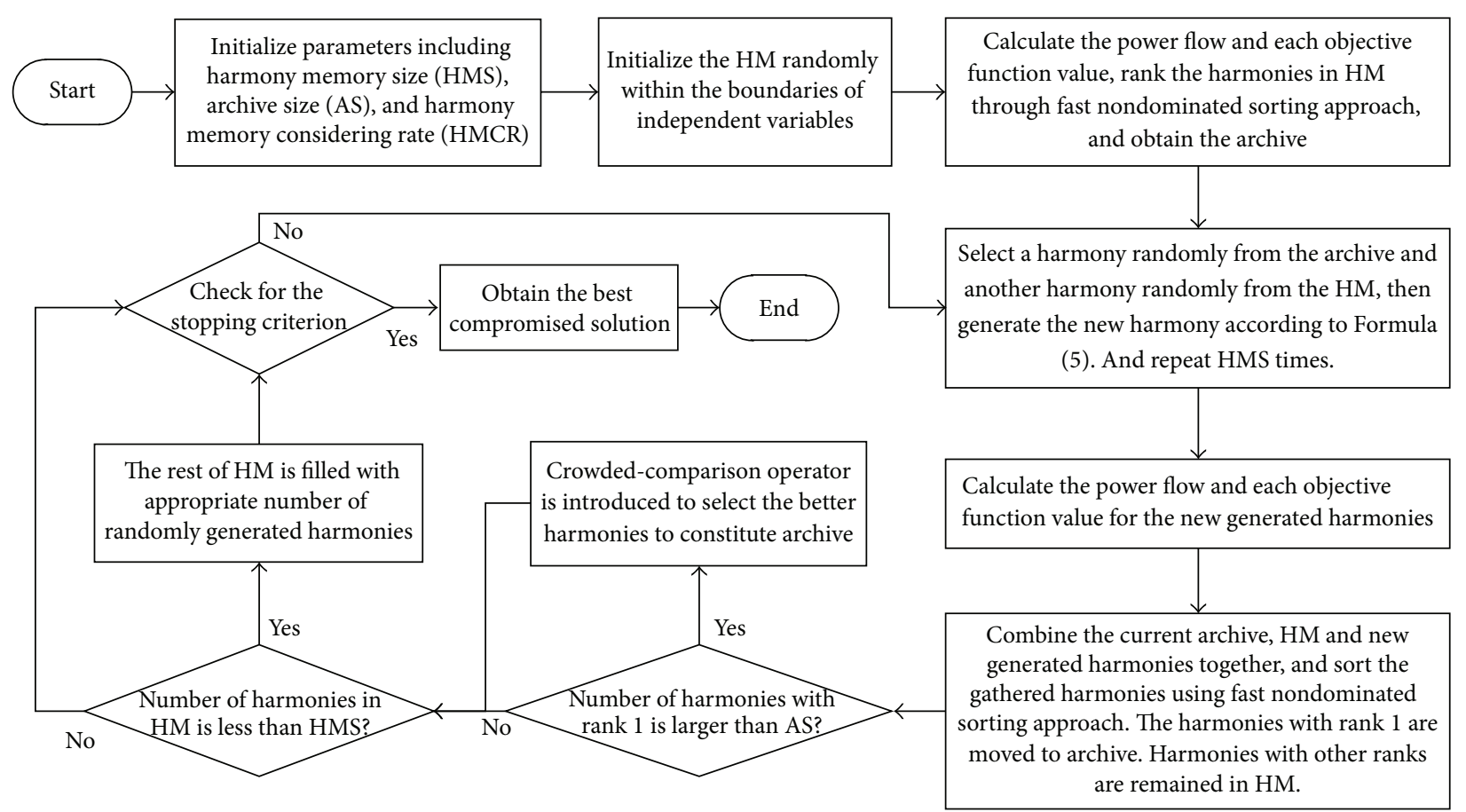

FIGURE 1: Flow chart of the proposed MOHS algorithm.

The inequality constraints can be described as follows:

(i) load bus voltage constraints:

$$
V_{i \min } \leq V_{i} \leq V_{i \max }
$$

(ii) thermal limits:

$\left|S_{i j}\right|=\left|V_{i}^{2} G_{i j}-V_{i} V_{j}\left(G_{i j} \cos \theta_{i j}+B_{i j} \sin \theta_{i j}\right)\right| \leq S_{i j}^{\max } ;$

(iii) generation output limits:

$$
\begin{aligned}
P_{\mathrm{DG} i}^{\min } & \leq P_{\mathrm{DG} i} \leq P_{\mathrm{DG} i}^{\max }, \\
Q_{\mathrm{DG} i}^{\min } & \leq Q_{\mathrm{DG} i} \leq Q_{\mathrm{DG} i}^{\max } ;
\end{aligned}
$$

(iv) DG penetration constraint:

$$
\sum_{i=1}^{n} P_{\mathrm{DG} i} \leq 0.25 S^{\mathrm{max}}, \quad\left(i \in \Phi_{S}\right),
$$

where $P_{\mathrm{DG} i}$ is the DG access capacity at bus $i$ and $S^{\max }$ is the maximum load capacity of distribution network.

4.1.3. Overview Formulation. Aggregating objectives and constraints, the problem can be formed as a nonlinear programming problem as follows:

$$
\begin{array}{ll}
\min & {\left[F_{1}(x), F_{2}(x), F_{3}(x)\right]} \\
\text { s.t. } & h_{i}(x)=\quad i=1,2, \ldots, n_{\mathrm{eq}} \\
& g_{i}(x) \leq 0 \quad i=1,2, \ldots, n_{\text {ineq }},
\end{array}
$$

where $x$ is the vector of independent variables, $n_{\mathrm{eq}}$ is the number of equality constraints, and $n_{\text {ineq }}$ is the number of inequality constraints. It is assumed that the DG has a prespecified power factor; and then there will be two control variables per DG installed, which can be shown as

$$
x^{T}=\left[\operatorname{Loc}_{\mathrm{DG}_{1}}, P_{\mathrm{DG}_{1}}, \ldots, \operatorname{Loc}_{\mathrm{DG}_{N_{\mathrm{DG}}}}, P_{\mathrm{DG}_{N_{-} \mathrm{DG}}}\right],
$$

where Loc $_{\mathrm{DG}}$ indicates the location of DG integration, $P_{\mathrm{DG}}$ indicates the active power output of DG, and N_DG is the maximal allowable injection number of DG.

4.2. Experiment Setting. To demonstrate the effectiveness of the proposed algorithm MOHS, the DG planning in the IEEE 33-bus test system is utilized as an example. The single line diagram of IEEE 33-bus system is shown in Figure 2, and its penetration rate is set as 25\%. Parameters used in MOHS are set as $\mathrm{MHS}=100, \mathrm{AS}=200$, and $\mathrm{HMCR}=0.9$. According to the experiments we have made, for the vast majority of distribution power system, the MOHS can obtain the Pareto solution set within 100 iterations. Without loss of generality, the maximum number of iteration in MOHS is set as 100 .

4.3. Optimization Results. At the last round of iteration in MOHS, the harmonies in archive compose the Pareto solution set of the optimization problem, which is shown in Figure 2. As displayed in Figure 2, each blue point represents a candidate solution in Pareto solution set. It shows that the MOHS has good global searching capability and the candidate solutions have uniform distribution. In addition, in order to demonstrate the advantages of MOHS to HS, the optimization results obtained by $\mathrm{HS}$ at different weights of 
objectives are also displayed in Figure 2. The red, brown, and green points represent the optimization solutions for cases $\mathrm{A}, \mathrm{B}$, and $\mathrm{C}$ which have massive differences among the three objective weights listed in Table 1, respectively. It shows that the three points are among the Pareto solution set of MOHS. It means that the candidate solutions of MOHS contain the optimized solutions in these three circumstances.

The optimization solution will be obtained by HS only if the weight for each objective is prespecified. And when the preference of decision maker is changed, the previously obtained solution is useless and the whole process of HS algorithm must be repeated again to get a new solution. For MOHS, when the weights of objectives are changed, the final solution can be extracted from the Pareto solution set using different weights, without necessity to obtain the Pareto solution set again. Compared with HS, the proposed MOHS has great convenience and rapidity for variable weight multiobjective optimization problem.

The best compromised solution and corresponding multiobjective function values of IEEE-33 system with and without DG units are illustrated in Table 2. As shown in Table 2, DG units are integrated to distribution power system at buses 17 and 32, namely, the end of each main feeder, with the size of $0.5137 \mathrm{MW}$ and $0.4248 \mathrm{MW}$, respectively. Compared with the case without DG, the integrated scheme has significant decrease in the three objective function values, which reduce $49.9 \%, 29.2 \%$, and $68.1 \%$ respectively. The effectiveness of solution obtained by MOHS can be demonstrated from the optimization results.

4.4. Algorithms Comparison. To demonstrate the global searching capability and convergence speed, the proposed MOHS is compared with the classic multiobjective evolution algorithm NSGA-II. Evaluation of multiobjective optimization algorithm is much complex than the single objective one; herein the $C$ metric [20] and outer solution [16] are utilized to illustrate the difference of optimization searching performance between MOHS and NSGA-II.

\subsubsection{Metric}

Definition 4. Let $X^{\prime}$ and $X^{\prime \prime}$ be two Pareto sets, the function $C$ represents the coverage extent of the ordered pair $\left(X^{\prime}, X^{\prime \prime}\right)$ in the interval $[0,1]$ :

$$
C\left(X^{\prime}, X^{\prime \prime}\right)=\frac{\left|\left\{a^{\prime \prime} \in X^{\prime \prime} ; \exists a^{\prime} \in X^{\prime}: a^{\prime} \prec a^{\prime \prime}\right\}\right|}{\left|X^{\prime \prime}\right|} .
$$

The value $C\left(X^{\prime}, X^{\prime \prime}\right)=1$ means that all solutions in $X^{\prime \prime}$ are dominated by solutions in $X^{\prime}$. The opposite condition, $C\left(X^{\prime}, X^{\prime \prime}\right)=0$, represents the situation that none of solutions in $X^{\prime \prime}$ are covered by the set $X^{\prime}$. Note that both $C\left(X^{\prime}, X^{\prime \prime}\right)$ and $C\left(X^{\prime \prime}, X^{\prime}\right)$ have to be considered, since $C\left(X^{\prime}, X^{\prime \prime}\right)$ is not necessarily equal to $1-C\left(X^{\prime \prime}, X^{\prime}\right)$.

Box-plot is a common tool to display distribution of statistical results, which is employed here to visualize the $C$ metric results of MOHS and NSGA-II. Each algorithm runs 30 times, respectively, and the $C$ metric results are demonstrated in Figure 3. As shown in Figure 3, the left box

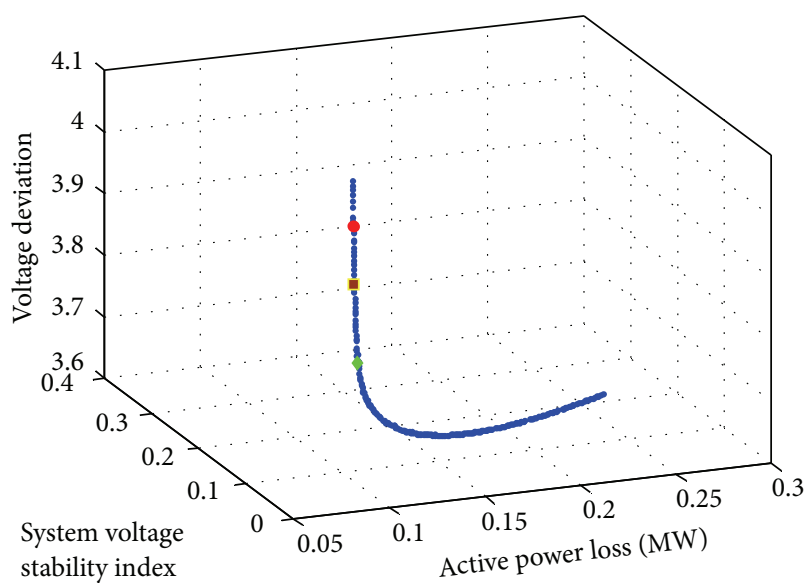

FIgure 2: The Pareto solution set of MOHS for IEEE 33-bus system.

TABLE 1: Optimization objective weights configuration.

\begin{tabular}{lccc}
\hline \multirow{2}{*}{ Case } & \multicolumn{3}{c}{ Optimization objective weights } \\
& 1st & 2nd & 3rd \\
\hline A & 0.8 & 0.1 & 0.1 \\
B & 0.1 & 0.8 & 0.1 \\
C & 0.1 & 0.1 & 0.8 \\
\hline
\end{tabular}

represents how many solutions of MOHS are dominated by solutions of NSGA-II, the right box represents how many solutions of NSGA-II are dominated by solutions of MOHS. The $C$ (NSGA-II, MOEA) has much lower distribution than the $C$ (MOEA, NSGA-II); namely, the solution set of MOEA has much better nondominated performance compared with NSGA-II. It shows that the proposed MOHS performs even better in finding the approximate Pareto-optimal front of feasible solutions.

4.4.2. Outer Solution Evolutionary Process. Outer solution is defined as the solution which has the minimum value for certain objective in Pareto-optimal front. It can be described as

$$
s_{l}^{(k)}=\left\{x_{i} \mid \forall x_{j} \in X^{(k)}: f_{l}\left(x_{i}\right) \leq f_{l}\left(x_{j}\right)\right\},
$$

where $s_{l}^{(k)}$ is the outer solution corresponding to $l$ th objective at the $k$ th iteration, $X^{(k)}$ is the solution set at the $k$ th iteration, and $f_{l}\left(x_{i}\right)$ is the $l$ th objective function value of solution $x_{i}$. The speed of optimization searching can be illustrated through analyzing the evolutionary process of outer solution.

The evolutionary process of outer solution for power loss is shown in Figure 4. The outer solutions for power loss converge to the same value after 15 iterations according to MOHS and NSGA-II. It means that the two algorithms achieve the approximate optimum after several iterations. However, MOHS shows better searching speed as it rapidly converges to optimum less than 10 iterations. The evolutionary process of outer solution for the other two objectives has the similar performance with power loss. Through demonstrating the 
TABLE 2: Result of IEEE 33-bus test system.

\begin{tabular}{lcccc}
\hline \multicolumn{2}{c}{ DG planning scheme } & & \multicolumn{2}{c}{ Multiobjective function values } \\
Position & Capacity (MW) & Power loss (MW) & Voltage stability index & Voltage deviation \\
\hline 17 & 0.5137 & 0.1016 & 0.0528 & 3.7405 \\
32 & 0.4248 & 0.2027 & 0.0746 & 11.7102 \\
\hline \multicolumn{2}{c}{ Without DG units } \\
\hline
\end{tabular}

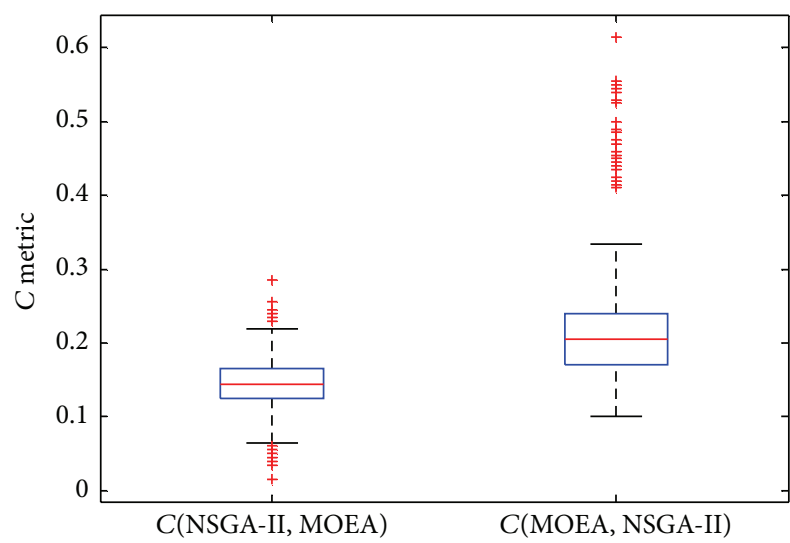

Figure 3: Box-plot of $C$ metric.

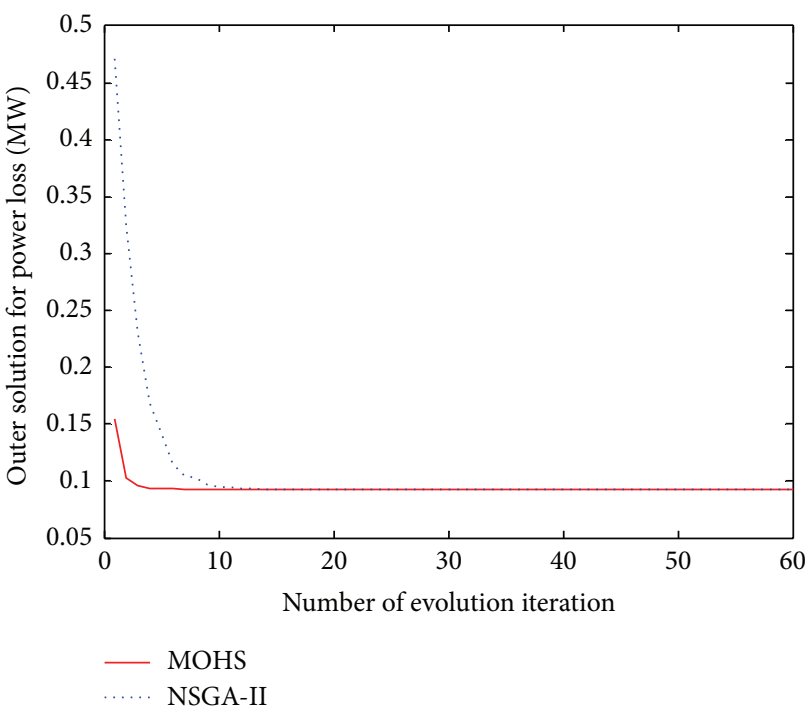

FIGURE 4: Evolutionary process of outer solution for power loss.

evolutionary process of outer solution, it is proved that the MOHS has a better performance in searching rapidity.

\section{Conclusions}

A novel intelligent searching algorithm, MOHS, is proposed with the combination of fast nondominated sorting in this paper. And the proposed MOHS is applied in the optimal placement and sizing of DG units in distribution power system. With consideration of minimal power loss, minimal voltage deviation, and maximal system voltage stability margin, the mathematical model of DG placement and sizing in power system can be formed as multiobjective optimization problem. IEEE 33-bus test system has been tested with the optimal DG integration planning using the proposed MOHS method. Through the metric comparison with NSGA-II, the MOHS can be demonstrated to have better performance.

\section{Conflict of Interests}

The authors declare that there is no conflict of interests regarding the publication of this paper.

\section{Acknowledgments}

This work was supported in part by the National Natural Science Foundation of China (Grant no. 51177152) and State Grid Corporation of China Research Program (Grant no. PD71-12010 ), and their financial support is much appreciated.

\section{References}

[1] C. Y. T. Ma, D. K. Y. Yau, N. K. Yip, N. S. V. Rao, and J. Chen, "Stochastic steepest descent optimization of multiple-objective mobile sensor coverage," IEEE Transactions on Vehicular Technology, vol. 61, no. 4, pp. 1810-1822, 2012.

[2] K.-Y. Liao, C.-Y. Chang, and J. C.-M. Li, "A parallel test pattern generation algorithm to meet multiple quality objectives," IEEE Transactions on Computer-Aided Design of Integrated Circuits and Systems, vol. 30, no. 11, pp. 1767-1772, 2011.

[3] D. Singh and K. S. Verma, "Multiobjective optimization for DG planning with load models," IEEE Transactions on Power Systems, vol. 24, no. 1, pp. 427-436, 2009.

[4] A. Saffar, R. Hooshmand, and A. Khodabakhshian, "A new fuzzy optimal reconfiguration of distribution systems for loss reduction and load balancing using ant colony search-based algorithm," Applied Soft Computing, vol. 11, no. 5, pp. 4021-4028, 2011.

[5] A. M. El-Zonkoly, "Optimal placement of multi-distributed generation units including different load models using particle swarm optimization," Swarm and Evolutionary Computation, vol. 1, no. 1, pp. 50-59, 2011.

[6] R. K. Singh and S. K. Goswami, "Optimum allocation of distributed generations based on nodal pricing for profit, loss reduction, and voltage improvement including voltage rise issue," International Journal of Electrical Power \& Energy Systems, vol. 32, no. 6, pp. 637-644, 2010.

[7] Z.-H. Zhan, J.-J. Li, J.-N. Cao, J. Zhang, H. S.-H. Chung, and Y.H. Shi, "Multiple populations for multiple objectives: a coevolutionary technique for solving multiobjective optimization 
problems," IEEE Transactions on Cybernetics, vol. 43, no. 2, pp. 445-463, 2013.

[8] Z. W. Geem, J. H. Kim, and G. V. Loganathan, "A new heuristic optimization algorithm: harmony search," Simulation, vol. 76, no. 2, pp. 60-68, 2001.

[9] S. Sivasubramani and K. S. Swarup, "Multi-objective harmony search algorithm for optimal power flow problem," International Journal of Electrical Power \& Energy Systems, vol. 33, no. 3, pp. 745-752, 2011.

[10] K. Nekooei, M. M. Farsangi, H. Nezamabadi-pour, and K. Y. Lee, "An improved multi-objective harmony search for optimal placement of DGs in distribution systems," IEEE Transactions on Smart Grid, vol. 4, no. 1, pp. 557-567, 2013.

[11] H. B. Puttgen, P. R. MacGregor, and F. C. Lambert, "Distributed generation: semantic hype or the dawn of a new era?" IEEE Power and Energy Magazine, vol. 1, no. 1, pp. 22-29, 2003.

[12] M. F. Shaaban, Y. M. Atwa, and E. F. El-Saadany, "DG allocation for benefit maximization in distribution networks," IEEE Transactions on Power Systems, vol. 28, no. 2, pp. 639-649, 2013.

[13] N. Khalesi, N. Rezaei, and M.-R. Haghifam, "DG allocation with application of dynamic programming for loss reduction and reliability improvement," International Journal of Electrical Power \& Energy Systems, vol. 33, no. 2, pp. 288-295, 2011.

[14] V. R. Pandi, H. H. Zeineldin, and W. Xiao, "Determining optimal location and size of distributed generation resources considering harmonic and protection coordination limits," IEEE Transactions on Power Systems, vol. 28, no. 2, pp. 12451254, 2013.

[15] K. Deb, A. Pratap, S. Agarwal, and T. Meyarivan, "A fast and elitist multiobjective genetic algorithm: NSGA-II," IEEE Transactions on Evolutionary Computation, vol. 6, no. 2, pp. 182197, 2002.

[16] E. Zitzler, M. Laumanns, and L. Thiele, "SPEA2: improving the strength Pareto evolutionary algorithm," TIK Report 103, Swiss Federal Institute of Technology (ETH), Zurich, Switzerland, 2001.

[17] M. A. Abido, "Multiobjective evolutionary algorithms for electric power dispatch problem," IEEE Transactions on Evolutionary Computation, vol. 10, no. 3, pp. 315-329, 2006.

[18] P. Kessel and H. Glavitsch, "Estimating the voltage stability of a power system," IEEE Transactions on Power Delivery, vol. 1, no. 3, pp. 346-354, 1986.

[19] G. B. Jasmon and L. H. C. C. Lee, "Distribution network reduction for voltage stability analysis and loadflow calculations," International Journal of Electrical Power \& Energy Systems, vol. 13, no. 1, pp. 9-13, 1991.

[20] E. Zitzler, K. Deb, and L. Thiele, "Comparison of multiobjective evolutionary algorithms: empirical results," Evolutionary Computation, vol. 8, no. 2, pp. 173-195, 2000. 


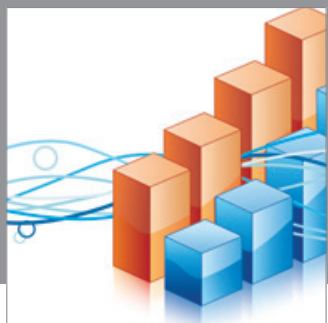

Advances in

Operations Research

mansans

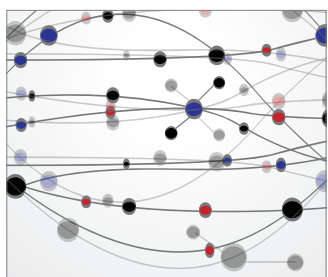

The Scientific World Journal
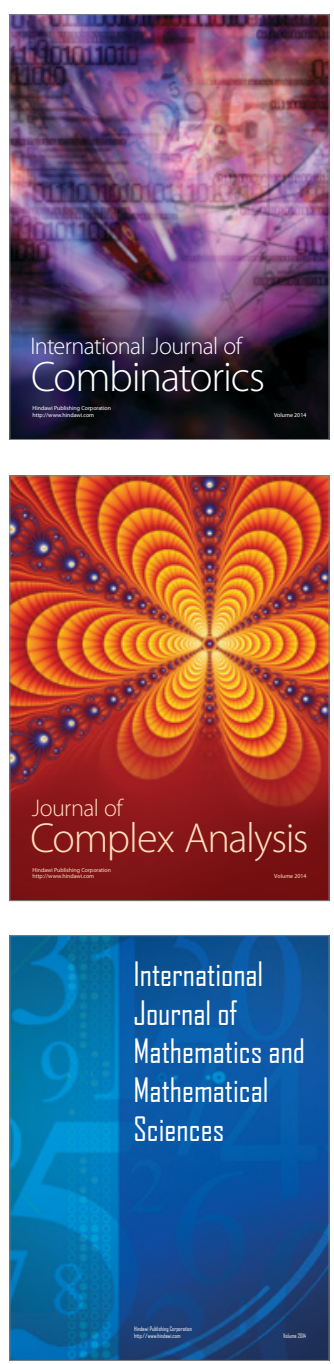
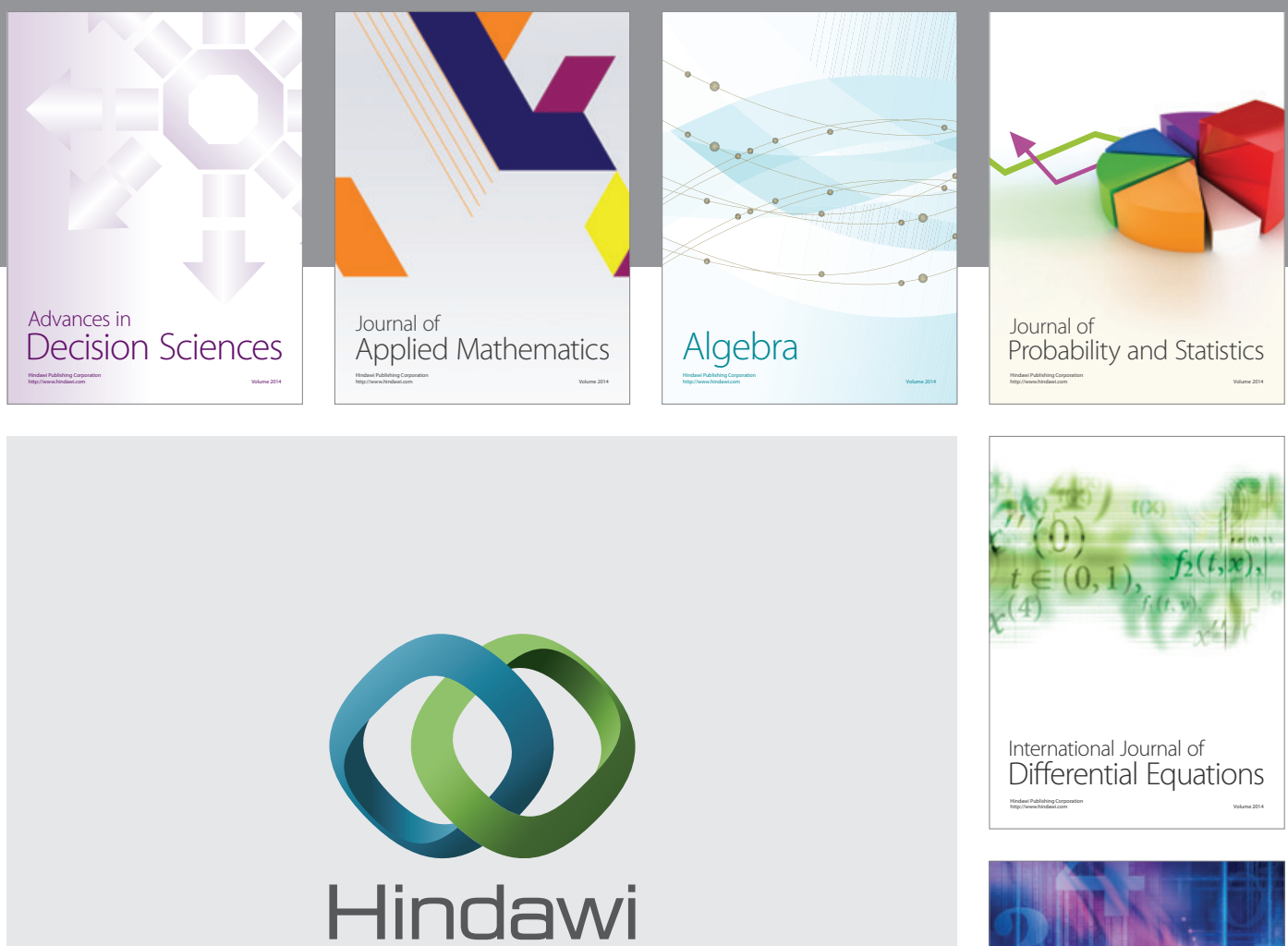

Submit your manuscripts at http://www.hindawi.com
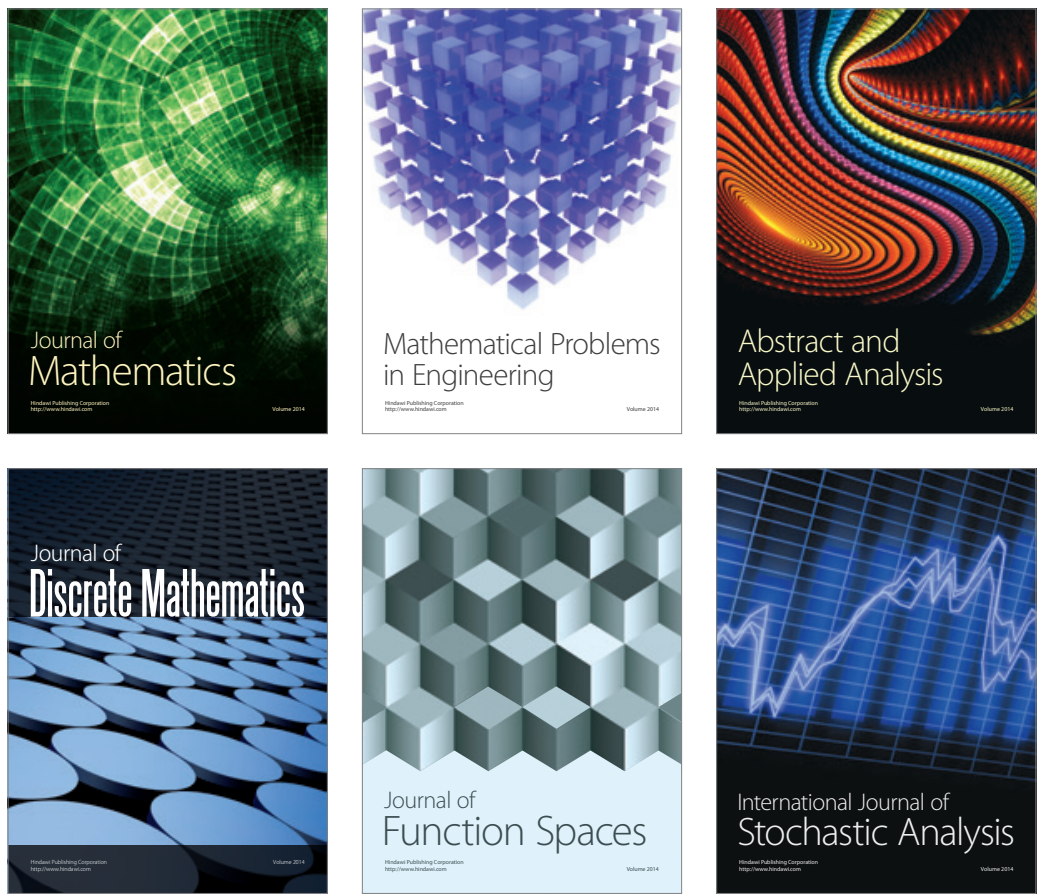

Journal of

Function Spaces

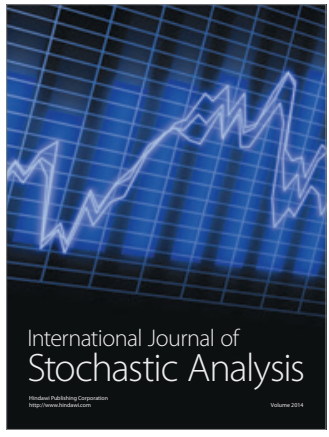

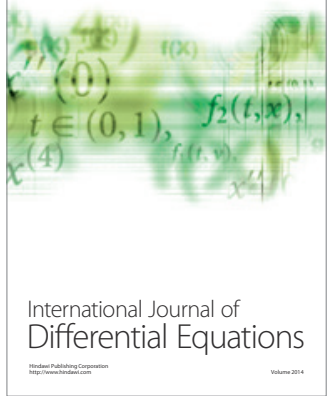
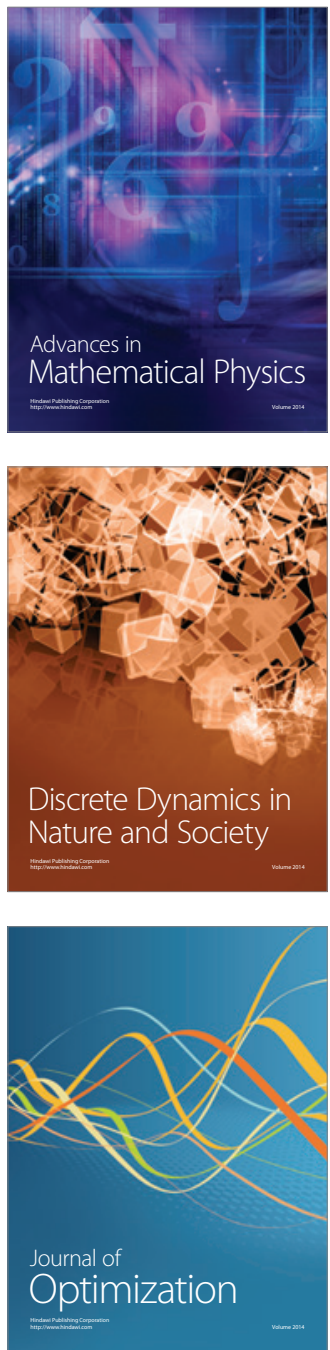